[Vũ1] Vũ Quôc Phóng, On the spectrum, complete trajectories, and asymptotic stability of linear semi-dynamical systems, J. Differential Equations 105 (1993), 30-45.

[Vü2] -, Semigroups with nonquasianalytic growth, Studia Math. 104 (1993), 229-241.

Ralph deLaubenfels

Scientia Research Institute

P.O. Box 988

Athens, Ohio 45701

U.S.A.

E-mail: 72260.2403@compuserve.com

Received October 20,1995

(3549)
Vũ Quôc Phóng Mathematics Department Ohio University Athens, Ohio 45701 U.S.A.

\section{On generalized Bergman spaces}

by

WOLFGANG LUSKY (Paderborn)

Abstract. Let $D$ be the open unit disc and $\mu$ a positive bounded measure on $[0,1]$. Extending results of Mateljevic/Panlovic and Shields/Williams we give Banach-space descriptions of the classes of all harmonic (holomorphic) functions $f: D \rightarrow \mathbb{C}$ satisfying $\int_{0}^{1}\left(\int_{0}^{2 \pi}\left|f\left(r e^{i \varphi}\right)\right|^{p} d \varphi\right)^{q / p} d \mu(r)<\infty$

1. Introduction. The aim of this paper is to give Banach space representations of certain classes of harmonic and holomorphic functions. Consider $D=\{z \in \mathbb{C}:|z|<1\}$ and put, for $0 \leq r$,

$$
M_{p}(f, r)=\left(\frac{1}{2 \pi} \int_{0}^{2 \pi}|f(r \exp (i \theta))|^{p} d \theta\right)^{1 / p} \quad \text { if } 1 \leq p<\infty,
$$

and $M_{\infty}(f, r)=\sup _{|z|=r}|f(z)|$.

We want to study harmonic functions $f: D \rightarrow \mathbb{C}$ which are not necessarily bounded but for which $M_{p}(f, r)$ grows in a controlled way as $r \rightarrow 1$. To this end we introduce a bounded (positive) measure $\mu$ on $[0,1]$ and put, for $1 \leq p \leq \infty$,

$$
\|f\|_{p, q}=\left(\int_{0}^{1} M_{p}^{q}(f, r) d \mu(r)\right)^{1 / q} \quad \text { if } 1 \leq q<\infty
$$

and

$$
\|f\|_{r, \infty}=\sup _{0 \leq r<1}\left(M_{p}(f, r) \mu([r, 1])\right) .
$$

We investigate the spaces

$$
\begin{aligned}
& b_{p, q}(\mu)=\left\{f: D=\left\{: \mathcal{C}: f \text { harmonic, }\|f\|_{p, q}<\infty\right\},\right. \\
& b_{p, 0}(\mu)=\left\{f \in b_{p, \infty}(\mu): \lim _{r \rightarrow+1} M_{p}(f, r) \mu([r, 1])=0\right\}
\end{aligned}
$$

and

$$
B_{p, q}(\mu)=\left\{f \in b_{p, q}(\mu): f \text { holomorphic }\right\} \quad \text { if } q=0 \text { or } 1 \leq q \leq \infty .
$$

1991. Mathematics Subject Classification: 46E15, 46B45. 
The assumption on the boundedness of $\mu$ is only used to make sure that these spaces contain all trigonometric polynomials and all polynomials, resp. If $\mu(\{1\})>0$ then our definitions, for $p=q$, yield the classical $L_{p^{-}}$and $H_{p}$-spaces which we want to exclude in what follows. So we assume

$$
\lim _{r \rightarrow 1} \mu([r, 1])=0 .
$$

If we have $\operatorname{supp} \mu \subset[0, a]$ for some $a<1$ then we can replace $[0,1]$ by $[0, a]$. Using substitution we see that it suffices to restrict ourselves to the case $a=1$, i.e.

$$
0<\mu([r, 1]) \quad \text { for each } r<1 .
$$

From now on we always assume (1.1) and (1.2).

EXAMPLE. Let $d \mu(r)=2 \pi r d r$. Then for $p=q<\infty$ we have $\|f\|_{p, q}=$ $\left(\iint_{D}|f(x+i y)|^{p} d x d y\right)^{1 / p}$. Hence in this case we obtain the classical Bergman spaces (see [1], [4], [10]).

For arbitrary $\mu$ put $v(r)=\mu([r, 1]) ; v$ is called a radial weight function. $B_{\infty, q}(\mu)$ and $b_{\infty, q}(\mu)$, for $q \in\{0, \infty\}$, are the weighted spaces considered in [11], [12], [14]-[17]. Note that, for any $1 \leq p \leq \infty$, we have $f \in$ $b_{p, \infty}(\mu)$ iff $M_{p}(f, r)=O(1 / v(r))$ as $r \rightarrow 1$. So, by characterizing $b_{p, \infty}(\mu)$ we obtain generalizations of results of Hardy and Littlewood ([8], [9], [5], Section 5, and Corollaries 2.6, 2.7 below). The space $b_{1,1}(\mu)$. was also considered in [15] and [16]. Our paper includes extensions of some results of Shields and Williams. We use non-trivial modifications of the methods of $[12]$.

Our main result states that, under a mild assumption on $\mu$, we have $b_{p, q}(\mu) \sim\left(\sum_{n} \oplus l_{p}^{n}\right)_{(q)}$. (" $\sim$ " means "is isomorphic to"). In this situation we can precisely determine for which measures $\mu$ we also have $B_{p, q}(\mu) \sim$ $\left(\sum_{n} \oplus l_{p}^{n}\right)_{(q)}$. For example this is false for all $q$ if $\mu=\sum_{n=1}^{\infty} \frac{1}{n(n+1)} \delta_{1-2^{-n}}$ and $p \in\{1, \infty\}$.

Our paper also extends the work of Mateljević and Pavlović [13], where in the case of analytic functions, $B_{p, q}(\mu) \sim\left(\sum_{n} \oplus l_{p}^{n}\right)_{(q)}$ was proved for $1<p<\infty$ for a more restricted class of measures $\mu$. (See also [3] and [20]. For another kind of representation in some special cases see [4].)

The paper is organized as follows. In Section 2 we state the main results; most of their proofs are given in Section 5 . In Section 4 we collect the Banach space properties of $\left(\sum \oplus l_{p}^{n}\right)_{(q)}$ and of related spaces needed for the proofs. Section 3 deals with elementary properties of trigonometric polynomials and the operators $R_{n}$, defined for a harmonic function $f(r \exp (i \varphi))=\sum_{k \in \mathbb{Z}} \alpha_{k} r^{|k|} \exp (i k \varphi)$ on $D$ as follows:
(1.3)

$$
\begin{aligned}
& \left(R_{n} f\right)(r \exp (i \varphi)) \\
& = \begin{cases}\sum_{|k| \leq 2^{n+1+1}} \alpha_{k} r^{|k|} \exp (i k \varphi) & \text { if } 1<p<\infty, \\
\sum_{|k| \leq 2^{n}} \alpha_{k} r^{|k|} \exp (i k \varphi \varphi) & \\
+\sum_{2^{n}+1 \leq|k| \leqslant 2^{n+1}} \frac{2^{n+1}-|k|}{2^{n}} \alpha_{k} r^{|k|} \exp (i k \varphi) & \text { if } p=1, \infty .\end{cases}
\end{aligned}
$$

(We put $R_{0}=0$.) Let

$\lambda_{p}=\sup s u p\left\{M_{n}\left(R_{n} f, r\right): f\right.$ a trigonometric polynomial, $\left.M_{p}(f, r) \leq 1\right\}$.

Since $R_{n}$ is a convolution operator with a Dirichlet or de la Vallée-Poussin kernel (sec [18], [19]), $\lambda_{p}$, does not depend on $r$ and we have $\left\|R_{n}\right\|_{p, q} \leq$ $\lambda_{p}\|f\|_{p, q}$ for all $f \in b_{p, q}(\mu)$. Morcover, we consider the Riesz projection

$$
(R f)(r \exp (i \varphi))=\sum_{k \geq 0} \alpha_{k} r^{|k|} \exp (i k \varphi) .
$$

$R$ is bounded for $\|\cdot\|_{p, q}$ if $1<p<\infty$ ([5], [18]).

We shall the the following convention. If not specified otherwise, $p$ is an element of $[1, \infty]$, and $q$ is an element of $\{0\} \cup[1, \infty]$. For Banach spaces $X_{n}$ put

$$
\begin{aligned}
& \left(\sum \oplus X_{n}\right)_{(a)}=\left\{\left(x_{n}\right): x_{n} \in X_{n} \text { for all } n,\left(\sum\left\|x_{n}\right\|^{q}\right)^{1 / q}<\infty\right\}, \\
& \left(\sum \oplus X_{n}\right)_{(\infty)}=\left\{\left(x_{n}\right): x_{n} \in X_{n} \text { for all } n, \sup _{n}\left\|x_{n}\right\|<\infty\right\}, \\
& \left(\sum \oplus X_{n}\right)_{(0)}=\left\{\left(x_{n}\right) \in\left(\sum \oplus X_{n}\right)_{(\infty)}: \lim _{n \rightarrow \infty}\left\|x_{n}\right\|=0\right\} .
\end{aligned}
$$

2. The main results. First we list some elementary properties of $B_{p, q}(\mu)$ and $b_{p, q}(k l)$.

2.1. Proposition. (a) All $B_{p, q}(\mu)$ and $b_{p, q}(\mu)$ are Banach spaces.

(b) Let $1 \leq q<\infty$ or $q$ to 0 . Then the trigonometric polynomials are dense in $b_{p, q}(\mu)$ whiles the polynomials are dense in $B_{p, q}(\mu)$.

Proof. (a) This follown from the fact that these spaces are closed in

$$
\left\{f: D \rightarrow \mathbb{C} \text { medsurable }: \int_{0}^{1}\left(\int_{0}^{2 \pi}\left|f\left(r e^{i \varphi}\right)\right|^{p} d \varphi\right)^{q / p} d \mu(r)<\infty\right\}
$$

if $1 \leq q<\infty$. The remaining cases $q=0, \infty$ can be proven similarly.

(b) For any $f \in b_{p_{1} q}(\mu)$ and $0<r<1$ we have $\lim _{n} M_{p}\left(f-R_{n} f, r\right)$ $=0$ in view of (1.3). This includes the case $p=\infty$ since $f$ is continuous 
on $D$. Moreover, $M_{p}\left(f-R_{n} f, r\right) \leq\left(1+\lambda_{p}\right) M_{p}(f, r)$. So using the dominated convergence theorem we see that $R_{n} \rightarrow$ id pointwise on $B_{p, q}(\mu)$ as well as on $b_{p, q}(\mu)$ if $1 \leq q<\infty$. If $q=0$ choose, for given $\varepsilon>0$, some $r_{0} \in[0,1[$ such that

$$
\left(1+\lambda_{p}\right) \sup _{r \geq r_{0}} M_{p}(f, r) \mu([r, 1]) \leq \varepsilon
$$

Then

$$
\left\|f-R_{n} f\right\|_{p, 0} \leq \max \left(\varepsilon, M_{p}\left(f-R_{n} f, r_{0}\right) \mu([0,1])\right)
$$

in view of the maximum principle. We also obtain $R_{n} \rightarrow \mathrm{id}$ (pointwise) in the case $q=0$. This implies (b).

2.2. Proposition. There are constants $a, b>0$ and, for every $p, q$, positive integers $m_{1}<m_{2}<\ldots$ such that for all $f \in b_{p, q}(\mu)$,

$a\left(\sum_{n}\left\|\left(R_{m_{n+1}}-R_{m_{n}}\right) f\right\|_{p, q}^{q}\right)^{1 / q} \leq\|f\|_{p, q} \leq b\left(\sum_{n}\left\|\left(R_{m_{n+1}}-R_{m_{n}}\right) f\right\|_{p, q}^{q}\right)^{1 / q}$

if $1 \leq q<\infty$ and

$$
a \sup _{n}\left\|\left(R_{m_{n+1}}-R_{m_{n}}\right) f\right\|_{p, q} \leq\|f\|_{p, q} \leq b \sup _{n}\left\|\left(R_{m_{n+1}}-R_{m_{n_{2}}}\right) f\right\|_{p, q}
$$

if $q=0$.

Proof. We deal with the case $1 \leq q<\infty$. The remaining case is similar (see [11]). Let $E_{n}$ be the span of all trigonometric polynomials of degree $\leq n$. Since the unit ball in $E_{n}$ is compact we obtain from (1.1),

$$
\lim _{s \rightarrow 1} \sup _{g \in E_{n} ;\|g\|_{p, q} \leq 1} \int_{s}^{1} M_{p}^{q}(g, r) d \mu(r)=0 .
$$

Moreover, we have $\left|\alpha_{k}\right|^{q} \int_{0}^{1} r^{|k| q} d \mu(r) \leq 1$ for any $f\left(r e^{i \varphi}\right)=\sum_{k} \alpha_{k} r^{|k|} e^{i k \varphi}$ with $\|f\|_{p, q} \leq 1$. Hence, by (1.3) and the Minkowski inequality,

$$
\begin{aligned}
\left(\int_{0}^{s} M_{p}^{q}\left(\left(\mathrm{id}-R_{n}\right) f, r\right) d \mu(r)\right)^{1 / q} & \leq \sum_{|k| \geq 2^{n^{n}}}\left|\alpha_{k}\right|\left(\int_{0}^{s} r^{|k| q} d \mu(r)\right)^{1 / q} \\
& \leq \sum_{|k| \geq 2^{n^{n}}}\left(\frac{\int_{0}^{s} r^{|k| q} d \mu(r)}{\int_{0}^{1} r^{|k| q} d \mu(r)}\right)^{1 / q} \\
& \leq \sum_{|k| \geq 2^{n}} \frac{s^{k}}{((1+s) / 2)^{k}}\left(\frac{\mu([0, s])}{\mu([(1+s) / 2,1])}\right)^{1 / q} .
\end{aligned}
$$

Thus, (1.2) yields, for every $s<1$,

$$
\lim _{n \rightarrow \infty} \sup _{\|f\|_{p, q} \leq 1} \int_{0}^{s} M_{p}^{q}\left(\left(\mathrm{id}-R_{n}\right) f, r\right) d \mu(r)=0 .
$$

Using induction and (2.1), (2.2) we find $s_{n}$ and $m_{n+1}$ with

$$
\begin{array}{r}
\sup _{\|f\|_{p, q} \leq 1} \int_{s_{n}}^{1} M_{p}^{q}\left(R_{m_{n}} f, r\right) d \mu(r) \leq 4^{-n-1} 3^{1-q}, \\
\sup _{\|f\|_{p, q} \leq 1} \int_{0}^{s_{n}} M_{p}^{q}\left(\left(\mathrm{id}-R_{m_{n+1}}\right) f, r\right) d \mu(r) \leq 4^{-n-1} 3^{1-q} .
\end{array}
$$

Now consider an arbitrary $f \in b_{p, q}(\mu)$ and put $f_{n}=\left(R_{m_{n+1}}-R_{m_{n}}\right) f$. We have $f=\sum f_{n}$ and $f_{n}+f_{n+1}=\left(R_{m_{n+2}}-R_{m_{n}}\right) f$. Using $(2.3)$ we obtain, for each $n$,

$$
\int_{s_{n}}^{s_{n+1}} M_{p}^{q}(f, r) d \mu(r) \leq 3^{q-1} \int_{s_{n}}^{s_{n+1}} M_{p}^{q}\left(f_{n}+f_{n+1}, r\right) d \mu(r)+\frac{2}{4^{n+1}}\|f\|_{p, q}^{q} .
$$

Summation yields

$$
\begin{aligned}
\|f\|_{p, q}^{q} & \leq 3^{q-1} \sum_{n} \int_{s_{n+1}}^{s_{n+1}} M_{p}^{q}\left(f_{n}+f_{n+1}, r\right) d \mu(r)+\frac{2}{3}\|f\|_{p, q}^{q} \\
& \leq 3^{q-\cdots \cdot 1} \sum_{n}\left\|f_{n}+f_{n+1,1}\right\|_{p, q}^{q}+\frac{2}{3}\|f\|_{p, q}^{q} .
\end{aligned}
$$

Using the Minkowski incquality we obtain the right-hand inequality of Proposition 2.2 .

Now (1.3) yiclds (id $\left.-R_{m_{n+1}}\right) f_{n}=f_{n}=R_{m_{n+2}} f_{n}$ (see (3.1), (3.2)). So, (2.3) applied to $f_{n} /\left\|f_{n}\right\|_{p, q}$ implies

$$
\int_{0}^{s_{n+\cdots}} M_{p}^{q}\left(f_{n}, r\right) d \mu(r) \leq 4^{-n-1} 3^{1-q}\left\|f_{n}\right\|_{p, q}^{q}
$$

and

$$
\int_{n+1,2}^{1} M_{p}^{q}\left(f_{n b}, r\right) d \mu(r) \leq A^{-n-n-1} 3^{1-q}\left\|f_{n}\right\|_{p, q}^{q}
$$

Hence:

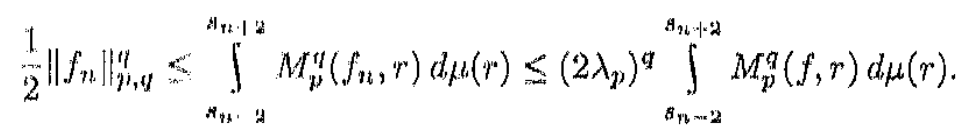

Summation yicldes $\frac{1}{2} \sum_{n}\left\|f_{n}\right\|_{p, q}^{q} \leq 4\left(2 \lambda_{p}\right)^{q}\|f\|_{p, q}^{q}$ and thus the left-hand inecquality of Proposition 2.2 .

2.3. COROLLARY. (q) The spaces $B_{p, q}(\mu)$ and $b_{p, q}(\mu)$ are reflexive if $1<q<\infty$.

(b) We have $B_{p, 0}(\mu)^{* * *}=B_{p_{1}, \infty}(\mu)$ and $b_{p, 0}(\mu)^{* * *}=b_{p, \infty}(\mu)$. 
Proof. Proposition 2.2 shows that $B_{p, q}(\mu)$ and $b_{p, q}(\mu)$ are isomorphic to subspaces of $\left(\sum \oplus X_{n}\right)_{(q)}$ for some finite-dimensional Banach spaces $X_{n}$. If $1<q<\infty$ the space $\left(\sum \oplus X_{n}\right)_{(q)}$ is reflexive. This yields (a). For the proof of $(\mathrm{b})$ observe that $\left(\sum \oplus X_{n}\right)_{(0)}^{* *}=\left(\sum \oplus X_{n}\right)_{(\infty)}$. Now it is very easy to see that the $w^{*}$-closures of $B_{p, 0}(\mu)$ and $b_{p, 0}(\mu)$ regarded as subspaces of $\left(\sum \oplus X_{n}\right)_{(0)}^{* *}$ are $B_{p, \infty}(\mu)$ and $b_{p, \infty}(\mu)$.

We want to improve Proposition 2.2 for a special class of measures.

2.4. Definition. Let $\mu$ be a bounded positive measure on $[0,1]$ satisfying (1.1) and (1.2). Put $\mu_{n}=\mu\left(\left[1-2^{-n}, 1\right]\right)$. We consider the following conditions:

$$
\begin{gathered}
\sup _{n}\left(\frac{\mu_{n}}{\mu_{n+1}}\right)<\infty, \\
\inf \limsup _{n \rightarrow \infty}\left(\frac{\mu_{n+k}}{\mu_{n}}\right)<1 .
\end{gathered}
$$

EXAMPLES. Put $d \mu_{1}(r)=(1-r)^{\alpha} d r$ for some $\alpha>-1, d \mu_{2}(r)=r^{\beta} d r$ for some $\beta>-1$,

$$
\begin{aligned}
d \mu_{3}(r) & =\frac{d r}{(1-r) \log ^{\gamma}(e /(1-r))} \quad \text { for some } \gamma>1, \\
\mu_{4} & =\sum_{k=1}^{\infty} \frac{1}{k(k+1)} \delta_{1-2^{-k}}
\end{aligned}
$$

Then $\mu_{1}, \mu_{2}$ satisfy $(\star)$ and ( $\left.\star \star\right)$ while $\mu_{3}, \mu_{4}$ fulfil $(\star)$ but not $(\star \star)$. $\mu_{1}$ was considered first by Hardy and Littlewood ([8], [9], see also [6], [7]). $\mu_{2}$ with $\beta=1$ yields the "classical" Bergman spaces [1].

2.5. THEOREM. Assume that $\mu$ satisfies $(\star)$. Then there are integers $I \leq m_{1}<m_{2}<\ldots$ and constants $a, b>0$ such that, for every $p, q$ and $f \in b_{p, q}(\mu)$, we have

$$
\begin{aligned}
& a\left(\sum_{n} M_{p}^{q}\left(\left(R_{m_{n}}-R_{m_{n-1}}\right) f, 1\right)\left(\mu_{m_{n}}-\mu_{m_{n+1}}\right)\right)^{1 / q} \\
& \quad \leq\|f\|_{p, q} \leq b\left(\sum_{n} M_{p}^{q}\left(\left(R_{m_{n}}-R_{m_{n-1}}\right) f, 1\right)\left(\mu_{m_{n}}-\mu_{m_{n+1}}\right)\right)^{1 / q}
\end{aligned}
$$

if $1 \leq q<\infty$ and

$$
\begin{aligned}
a \sup _{n} M_{p}\left(\left(R_{m_{n}}-\right.\right. & \left.\left.R_{m_{n-1}}\right) f, 1\right) \mu_{m_{n}} \\
& \leq\|f\|_{p, q} \leq b \sup _{n} M_{p}\left(\left(R_{m_{n}}-R_{m_{n-1}}\right) f, 1\right) \mu_{m_{n}}
\end{aligned}
$$

if $q \in\{0, \infty\}$. If $(\star \star)$ holds then we can choose $m_{n}=K n$ for some integer $K$. If ( $\star \star)$ is not satisfied and $p \in\{1, \infty\}$ then for any sequence $\left(m_{n}\right)$ with (2.4) or (2.5) we have $\sup _{n}\left(m_{n}-m_{n-1}\right)=\infty$.
Remark. Recall that $R_{m} f$ is a trigonometric polynomial, hence $M_{p}\left(R_{m} f, 1\right)$ makes sense. The proof of the theorem as well as of the following corollaries will be given in Section. 5 . The proof shows that we can choose the $m_{n}$ by induction such that $m_{1}=1$ and $m_{n+1}$ is the smallest integer larger than $m_{n}$ with $\mu_{m_{\mathrm{n}}} \geq 3 \mu_{m_{n+1}+1}$.

In [3] and [13] measures of the form $d \mu(r)=(1-r)^{-1} \varphi(1-r) d r$ were considered where $\varphi$ is a non-decreasing function satisfying two further conditions which imply $(\star)$ and $(* \star)$. Hence Theorem 2.5 includes, for $q \geq 1$, Theorem 2.1.(b) of [13] and Corollary 1 of [3].

Consider a harmonic function $f: \underset{\sim}{D} \rightarrow \mathbb{C}$ and let $\tilde{f}$ be its trigonometric conjugate, i.e. the harmonic function $\tilde{f}$ with $\widetilde{f}(0)=0$ such that $\operatorname{Re} f+i \operatorname{Re} \widetilde{f}$ and $\operatorname{Im} f+i \operatorname{Im} \tilde{f}$ are holomorphic. We obtain

(2.6) $\tilde{f}=-i R f+i(\mathrm{id}-R) f+i f(0)$ and $R f=\frac{1}{2}(f+i \widetilde{f})+\frac{1}{2} f(0)$.

2.6. Corollary. Let $\mu$ satisfy $(*)$.

(a) $b_{p, q}(\mu) \sim\left(\sum_{n} \oplus l_{p}^{n}\right)_{(q)}$ for all $p$ and $q$.

(b) $B_{p, q}(\mu) \sim\left(\sum_{n} \oplus l_{p}^{n}\right)_{(q)}$ for all $q$ and $1<p<\infty$.

(c) If $1<p<\infty$ and $q$ is arbitrary then the Riesz projection is a bounded operator from $b_{p, q}(\mu)$ onto $B_{p, q}(\mu)$.

(d) Let $1<p<\infty$, let $q$ be arbitrary and consider a harmonic function $f: D \rightarrow \mathbb{C}$. Then $\|f\|_{p, q}<\infty$ if and only if $\|\widetilde{f}\|_{p, q}<\infty$.

For the remaining casces there are some notable exceptions.

2.7. Corollary. Assume that $(\star)$ holds. Let $q$ be arbitrary and $p \in$ $\{1, \infty\}$. Then the following are equivalent:

(i) $B_{p, q}(\mu) \sim\left(\sum_{n}(\Theta)_{p}^{n}\right)_{(q)}$.

(ii) $R$. is a bounded operator from $b_{p, q}(\mu)$ onto $B_{p, q}(\mu)$.

(iii) H. satisfiets $(* *)$.

(iv) For a harmontic function $f: D \rightarrow \mathbb{C}$ we have $\|f\|_{p, q}<\infty$ if and only if $\|\tilde{f}\|_{p, q}<\infty$.

Remark. 2.5. 2.7 cxtend the results of [12] where the cases $p=\infty$ and $q \in\{0, \infty\}$ wore proved. Corollary 2.7 gives a positive answer to a problem raiserl in [13], p. 236. (This problem was independently solved by Wojtasyczylk in [20].) For $d_{1} \omega_{2}(r)=r d r$ we obtain the known isomorphic represcntations $b_{1, p}\left(\mu_{2}\right) \sim B_{p, p}\left(\mu_{2}\right) \sim l_{p}([10]$, [16]). However, for the measures $\mu_{33}$ and $\mu_{4}$ of the above exarnples we have $B_{p, q}(\mu) \nsim\left(\sum \oplus l_{p}^{n}\right)_{(q)}$ if $p \in\{1, \infty\}$ (in particular, $\left.B_{1,1}(\mu) \not l_{1}\right)$ but $B_{p, \infty}(\mu l) \sim\left(\sum \oplus l_{p}^{n}\right)_{(\infty)}$ whenever $1<p<\infty$. 
Corollary 2.7(iv) together with Corollary 2.6(d) might be regarded as a generalization of some Hardy-Littlewood theorems: Considering

$$
d \mu_{1}(r)=(1-r)^{\alpha} d r, \quad \alpha>-1, \quad \text { and } \quad q=\infty
$$

we obtain, as $r \rightarrow 1$,

$$
M_{p}(f, r)=O\left(\frac{1}{(1-r)^{\alpha+1}}\right) \text { if and only if } M_{p}(\widetilde{f}, r)=O\left(\frac{1}{(1-r)^{\alpha+1}}\right)
$$

([5], Theorem 5.7).

Virtually everything carries over to the case where $D$ is the Euclidean ball in $\mathbb{C}^{n}$.

3. Trigonometric polynomials. Here we collect some basic properties of the operators $R_{n}$. Clearly, we always have

$$
R_{n} R_{m}=R_{\min (n, m)} \quad \text { if } n \neq m \text {. }
$$

Sometimes we use the following consequence of (3.1):

$$
\left(R_{q}-R_{p}\right)\left(R_{n}-R_{m}\right)= \begin{cases}R_{n}-R_{m} & \text { if } p<m<n<q, \\ R_{q}-R_{p} & \text { if } m<p<q<n, \\ 0 & \text { if } q>p>n>m \\ & \text { or } n>m>q>p .\end{cases}
$$

For $f\left(r e^{i \varphi}\right)=\sum \alpha_{k} r^{|k|} e^{i k \varphi}$ put

$$
\left(\sigma_{m} f\right)\left(r e^{i \varphi}\right)=\sum_{|k| \leq m} \frac{m-|k|}{m} \alpha_{k} r^{|k|} e^{i k \varphi} .
$$

Then $\sigma_{m}$ is contractive with respect to $M_{p}(\cdot, r)([18])$.

3.1. LEMMA. There is a universal constant $c>0$ such that for $p \in\{1, \infty\}$ and all $r>0$ we have

(3.4) $\quad M_{p}\left(R\left(R_{n+1}-R_{n}\right) f, r\right) \leq c M_{p}\left(\left(R_{n+1}-R_{n}\right) f, r\right), \quad n=1,2, \ldots$, whenever $f$ is a harmonic function.

Proof. For each $m$ we have, in view of (1.3),

$$
R\left(R_{m+1}-R_{m}\right) f=e^{i 2^{m+1} \varphi} \sigma_{2^{m+1}}\left(e^{-i 2^{m+1} \varphi} f\right)-\frac{1}{2} e^{i 2^{m} \varphi} \sigma_{2^{m}}\left(e^{-i 2^{m} \varphi} f\right) .
$$

We conclude that $M_{p}\left(R\left(R_{n+2}-R_{n-1}\right) f, r\right) \leq \frac{9}{2} M_{p}(f, r)$. Replacing $f$ by $\left(R_{n+1}-R_{n}\right) f$ yields easily (3.4) (see [12], Corollary 3.1).

3.2. Lemma. Let $p \in\{1, \infty\}$. Consider integers $1 \leq m_{j}<n_{j}$ with $\sup _{j}\left(n_{j}-m_{j}\right)=\infty$. Then for any $\beta>0$ there are a trigonometric polynomial $f: D \rightarrow \mathbb{C}$ and an integer $k$ with $\left(R_{n_{k}}-R_{m_{k}}\right) f=f$ such that

$$
M_{p}(f, 1)=1-\text { but } M_{p}(R f, 1)>\beta \text {. }
$$

Proof. For $p=\infty$ this is essentially [12], Lemma 3.5: Put $h\left(r e^{i \varphi}\right)=$ $\sum_{j=1}^{\infty} \frac{1}{j} r^{j} \sin (j \varphi)$ which is the harmonic extension of $h\left(e^{i \varphi}\right)=i(\pi-\varphi), 0 \leq$ $\varphi<2 \pi$. For every integer $k$ with $n_{k} \geq m_{k}+3$ define $f_{k}=\left(R_{n_{k}-1}-R_{m_{k}+1}\right) h$. Then $M_{\infty}\left(f_{k}, 1\right) \leq 2 \lambda_{\infty} \pi$ and, in view of $(1.3)$

$$
M_{\infty}\left(R f_{k}, 1\right) \geq \sum_{j=2^{2 m_{k}+2}}^{2^{n_{k}-1}} \frac{1}{j}
$$

Since $\sup _{k}\left(n_{k}-m m_{k}-3\right)=\infty$ we find $k$ such that $M_{\infty}\left(R f_{k}, 1\right)>2 \lambda_{\infty} \pi \beta$. By (3.2), $f:=f_{k} / M_{(\infty)}\left(f_{k}, 1\right)$ proves the case $p=\infty$.

Since $\sup \left(n_{k}-m_{k}-4\right)=\infty$ we also find a trigonometric polynomial $d$ and an integer $k$ with

$$
\left(R_{n_{k}-2}-R_{m_{k_{0}+2}}\right) d=d, \quad M_{\infty}(d, 1)=1 \text { and } M_{\infty}(R d, 1)>2 \lambda_{1} \beta .
$$

Consider a harmonic $g$ with $M_{1}(g, 1)=1$ and

$$
\frac{1}{2 \pi} \int_{0}^{2 \pi} g\left(e^{i \varphi}\right) \cdot(R d)\left(e^{-i \varphi}\right) d \varphi>2 \lambda_{1} \beta
$$

Since $\left(R_{n_{k-1}-1}-R_{m_{k+1}+1}\right)^{*}=R_{n_{k j}-1}-R_{m_{k}+1}$ we obtain, according to $(3.2)$, $M_{1}\left(R\left(R_{n_{k+1}-1}-R_{m_{k+1}+1}\right) g, 1\right)>2 \lambda_{1} \beta$. Moreover, $M_{1}\left(\left(R_{n_{k}-1}-R_{m_{k}+1}\right) g, 1\right)$ $\leq 2 \lambda_{1}$. By (3.2), $f:=\left(R_{n_{k}-1}-R_{m_{k}+1}\right) g / M_{1}\left(\left(R_{n_{k}-1}-R_{m_{k}+1}\right) g, 1\right)$ proves the case $p=1$.

3.3. LIGMMA. Let $0<r<s$.

(a) If $f$ is a trigonometric polynomial of degree $n$ then

$$
M_{p}(f, s) \leq(s / r)^{2 n} M_{p}(f, r) .
$$

(b) Let $f\left(t e^{i \varphi}\right)=\sum_{|k| \geq n_{b}} \alpha_{k} t^{|k|} e^{i k \varphi}$ for some integer $m>0$. Then

$$
M_{\eta}(f, r) \leq c(r / s)^{m} M_{p}(f, s)
$$

for some: universal constant a which does not depend on $f, m, r$ or $s$.

Proof. (a) Let $1 \leq p<\infty$. We may assume $r \leq s<\exp (1 /(2 n)) r$ (if the lemma holds for these $r$ and $s$ then repeated application yields the general caste). Let $f\left(r e^{i / q}\right)=\sum_{|k| \leqslant n} \alpha_{k} r^{|k|} e^{i k \varphi}$. Fix $z \in \partial D$. Then (3.3) yields

$$
\left(\frac{n}{t}\left(\mathrm{id}-\sigma_{n}\right) f\right)(z)=\sum_{0<|k| \leq n}|k| \alpha_{k} t^{|k|-1} z^{k}
$$

This implies, with $1 / p^{\prime}+1 / p=1$, 


$$
\begin{aligned}
M_{p}(f, s)- & M_{p}(f, r) \\
& \leq\left(\frac{1}{2 \pi} \int_{0}^{2 \pi}\left|f\left(s e^{i \varphi}\right)-f\left(r e^{i \varphi}\right)\right|^{p} d \varphi\right)^{1 / p} \\
& =\left(\frac{1}{2 \pi} \int_{0}^{2 \pi}\left|\int_{r}^{s}\left(\frac{n}{t}\left(\mathrm{id}-\sigma_{n}\right) f\right)\left(t e^{i \varphi}\right) d t\right|^{p} d \varphi\right)^{1 / p} \\
& \leq n\left(\frac{1}{2 \pi} \int_{0}^{2 \pi}\left(\int_{r}^{s} \frac{d t}{t}\right)^{p / p^{\prime}}\left(\int_{r}^{s}\left|\left(\left(\mathrm{id}-\sigma_{n}\right) f\right)\left(t e^{i \varphi}\right)\right|^{p} \frac{d t}{t}\right) d \varphi\right)^{1 / p}
\end{aligned}
$$$$
\leq 2 n M_{p}(f, s) \log (s / r) \text {. }
$$

Hence $M_{p}(f, s) \leq(1-2 n \log (s / r))^{-1} M_{p}(f, r)$. For a fixed integer $m$ put $r_{j}=r^{(m-j) / m} s^{j / m}, j=0, \ldots, m$. Then we have $r_{j+1} / r_{j}=(s / r)^{1 / m}$ and $r_{j} \leq r_{j+1}<e^{1 /(2 n)} r_{j}$. Repeated application of what we have just proved yields

$$
\begin{aligned}
M_{p}(f, s) & \leq\left(1-\frac{2 n}{m} \log \left(\frac{s}{r}\right)\right)^{-1} M_{p}\left(f, r_{m-1}\right) \leq \ldots \\
& \leq\left(1-\frac{2 n}{m} \log \left(\frac{s}{r}\right)\right)^{-m} M_{p}(f, r) .
\end{aligned}
$$

If $m \rightarrow \infty$ then $(1-(2 n / m) \log (s / r))^{-m}$ tends to $\exp (2 n \log (s / r))=$ $(s / r)^{2 n}$. This proves the case $1 \leq p<\infty$. The proof for $p=\infty$ is the same.

(b) It suffices to assume $r / s \leq 1-1 / m$. (For $r / s>1-1 / m$ we have $M_{p}(f, r) \leq 2 e(r / s)^{m} M_{p}(f, s)$.) The inequality of (b) is clear if $f$ is holomorphic (even with $c=1$ ). For arbitrary $f$ satisfying the assumption let $k$ be such that

$$
2^{k+1} \leq m \leq 2^{k+2} .
$$

Then we have $\sum_{j=0}^{\infty}\left(R_{k+j+1}-R_{k+j}\right) f=f$. Put $f_{1}=R\left(R_{k+1}-R_{k}\right) f$ and $f_{2}=($ id $-R)\left(R_{k+1}-R_{k}\right) f$. Using Lemma 3.1 and the continuity of $R$ if $1<p<\infty$ we find a universal constant $c_{1}>0$ with

$$
\begin{aligned}
M_{p}\left(\left(R_{k+1}-R_{k}\right) f, r\right) & \leq M_{p}\left(f_{1}, r\right)+M_{p}\left(f_{2}, r\right) \\
& \leq(r / s)^{m}\left(M_{p}\left(f_{1}, s\right)+M_{p}\left(f_{2}, s\right)\right) \\
& \leq c_{1}(r / s)^{m} M_{p}\left(\left(R_{k+1}-R_{k}\right) f, s\right) \\
& \leq 2 \lambda_{p} c_{1}(r / s)^{m} M_{p}(f, s) .
\end{aligned}
$$

Similarly,

$$
M_{p}\left(\left(R_{k+j+1}-R_{k+j}\right) f, r\right) \leq 2 \lambda_{p} c_{1}(r / s)^{2^{k+j}} M_{p}(f, s), \quad j=1,2, \ldots,
$$

since $\left(R_{k+j+1}-R_{k+1+j}\right) f$ is spanned by $\bar{z}^{2^{k+j}}, z^{2^{k+j}}, \ldots, \bar{z}^{2^{k+j+1}}, z^{2^{k+j+1}}$. Hence for $r / s \leq 1-1 / m$ we obtain

$$
\begin{aligned}
M_{p}(f, r) & \leq 2 \lambda_{p} c_{1}(r / s)^{m}\left(1+\sum_{j=1}^{\infty}(r / s)^{2^{k+j}-m}\right) M_{p}(f, s) \\
& \leq 2 \lambda_{p} c_{1}(r / s)^{m}\left(1+\sum_{j=1}^{\infty} \exp \left(-\frac{2^{k+j}-m}{m}\right)\right) M_{p}(f, s) .
\end{aligned}
$$

In view of (3.5) there is a universal constant $c>0$ with

$$
M_{p}(f, r) \leq c(r / s)^{m} M_{p}(f, s)
$$

4. The Banach spaces $\left(\sum \oplus l_{p}^{n}\right)_{(q)}$. Let $d(\cdot, \cdot)$ be the Banach-Mazur distance between two Banach spaces.

4.1. Lemma. Put $X=\left(\sum \oplus l_{p}^{n}\right)_{(q)}$. Let $n_{k}$ be a sequence of positive integers with, $\sup _{k} n_{k}=\infty$. Then

$$
\left(\sum_{k=1}^{\infty} \oplus l_{p}^{n_{k}+k}\right)_{(q)} \sim X \sim(X \oplus X \oplus \ldots)_{(q)} .
$$

Proof. For cach integer $m>0$ find $n_{k}>m$. We obtain

$$
d\left(l_{p}^{n_{k}},\left(l_{p}^{m} \oplus l_{p}^{n_{k}-m}\right)_{(q)}\right) \leq 2 \text {. }
$$

Hence there is a set $N$ of integers $n_{k}-m$ with

$$
d\left(\left(\sum \oplus l_{p}^{n_{b}}\right)_{(q)},\left(\left(\sum_{m=1}^{\infty} \oplus l_{p}^{m}\right) \oplus\left(\sum_{j \in N} \oplus l_{p}^{j}\right)\right)_{(q)}\right) \leq 2 .
$$

Moreover, in the same way, for any infinite subset $N_{m}$ of positive integers we find integers $m_{k}$, with

$$
\left.d\left(\left(\sum_{j \in N_{m b}} \oplus l_{p}^{j}\right)_{(q)}\right)^{k}\left(\left(l_{p}^{m} \oplus l_{p}^{m} \oplus \ldots\right) \oplus\left(\sum_{k} \oplus l_{p}^{m_{k}}\right)\right)_{(q)}\right) \leq 2
$$

If we split the positive integers into a sequence of disjoint infinite subsets $N_{m}$ them (4.2) shown that $d\left(X,(X \oplus X \oplus \ldots)_{(q)}\right) \leq 2$. This together with (4.1) yieldis

$$
\left(\sum_{k=1}^{\infty} \oplus l_{p}^{n_{k}}\right)_{(q)} \sim\left((X \oplus X \oplus \ldots) \oplus\left(\sum_{j \in N} \oplus l_{p}^{j}\right)\right)_{(q)} \sim X .
$$

Next, consicler $\alpha_{k}>0$ such that

$$
0<\inf \left(\frac{\alpha_{k}}{\alpha_{k+1}}\right) \leq \sup \left(\frac{\alpha_{k}}{\alpha_{k+1}}\right)<\infty
$$


Furthermore, take integers $m_{0}=0<m_{1}<m_{2}<\ldots$ and define, for harmonic $f$

$$
\|f\|_{p, q}= \begin{cases}\left(\sum_{k} M_{p}\left(\left(R_{m_{k}}-R_{m_{k-1}}\right) f, 1\right)^{q} \alpha_{k}\right)^{1 / q} & \text { if } q \notin\{0, \infty\} \\ \sup _{k} M_{p}\left(\left(R_{m_{k}}-R_{m_{k-1}}\right) f, 1\right) \alpha_{k} & \text { if } q \in\{0, \infty\}\end{cases}
$$

Let

$$
\begin{aligned}
& Z_{p, q}=\left\{f: D \rightarrow \mathbb{C}: f \text { harmonic, }\|f\|_{p, q}<\infty\right\} \quad \text { for } q \neq 0, \\
& Z_{p, 0}=\left\{f \in Z_{p, \infty}: \lim _{n} M_{p}\left(\left(R_{m_{n}}-R_{m_{n-1}}\right) f, 1\right) \alpha_{n}=0\right\}, \\
& Y_{p, q}=\left\{f \in Z_{p, q}: f \text { holomorphic }\right\} .
\end{aligned}
$$

4.2. Lemma. Let $N$ be a positive integer. Then each $Y_{p, q}$ contains a subspace $X$ with a projection $Q: Z_{p, q} \rightarrow X$ such that

$$
d\left(X,\left(\sum_{j} \oplus l_{p}^{j}\right)_{(q)}\right) \leq 2, \quad\|Q\| \leq 2 \quad \text { and } R_{N} f=0 \text { for all } f \in X .
$$

Proof. Put $F_{k}=\operatorname{span}\left\{z^{j}: 2^{m_{k-1}+1} \leq j \leq 2^{m_{k}-1}\right\}$ if $m_{k-1}+1<m_{k}-1$. In view of (3.2) we obtain

$$
\text { (4.4) } \quad\left(R_{m_{j}}-R_{m_{j-1}}\right) f=\left\{\begin{array}{ll}
f & \text { if } j=k \\
0 & \text { else }
\end{array} \quad \text { for all } f \in F_{k} .\right.
$$

Since by assumption $\sup _{k} \operatorname{dim} F_{k}=\infty$ we find, for each $j$, a suitable $k_{j}$, a subspace $E_{k_{j}} \subset F_{k_{j}}$ with $d\left(E_{k_{j}}, l_{p}^{j}\right) \leq 2$ and a projection $P_{k_{j}}: L_{p}(\partial D) \rightarrow$ $E_{k_{j}}$ with $\left\|P_{k_{j}}\right\| \leq 2$. Here we consider the norm $M_{p}(g, 1) \alpha_{k_{j}}^{1 / q}$ on $L_{p}(\partial D)$ which coincides with $\|\cdot\|_{p, q}$ on $F_{k_{j}}$ by (4.4). (Of course $E_{k_{j}}$ and $P_{k_{j}}$ exist. At first consider the norm $M_{p}(g, 1)$ on $L_{p}(\partial D)$. Find a complemented subspace $E \subset L_{p}(\partial D)$ with $d\left(E, l_{p}^{j}\right) \leq 2$ consisting of trigonometric polynomials. Then apply a shift into a suitable $F_{k}$ which is possible since $\sup _{k}\left(2^{m_{k}-1}-\right.$ $\left.2^{m_{k-1}+1}\right)=\infty$. In particular, if $k$ is large enough we have $R_{N} \mid E_{k}=0$. Everything remains true if we go over to the norm $M_{p}(g, 1) \alpha_{k}^{1 / q}$.)

For $k \neq k_{j}, j=1,2, \ldots$, put $P_{k}=0$ and $E_{k}=\{0\}$. Let

$$
X=\left\{f \in Y_{p, q}:\left(R_{m_{k}}-R_{m_{k-1}}\right) f \in E_{k} \text { for all } k\right\} .
$$

According to the definition of the norm $\|\cdot\|_{p, q}$ and (4.4) we obtain

$$
d\left(X,\left(\sum \oplus l_{p}^{j}\right)_{(q)}\right) \leq 2
$$

Finally, put $Q f=\sum_{k} P_{k}\left(R_{m_{k}}-R_{m_{k-1}}\right) f$. Then we have, by (4.4), if $q \neq 0, \infty$

$$
\begin{aligned}
\|Q f\|_{p, q} & =\left(\sum_{k} M_{p}\left(P_{k}\left(R_{m_{k}}-R_{m_{k-1}}\right) f, 1\right)^{q} \alpha_{k}\right)^{1 / q} \\
& \leq 2\left(\sum_{k} M_{p}\left(\left(R_{m_{k}}-R_{m_{k-1}}\right) f, 1\right)^{q} \alpha_{k}\right)^{1 / q}=2\|f\|_{p, q} .
\end{aligned}
$$

(4.4) also shows that $Q$ is a projection. The proof for $q \in\{0, \infty\}$ is the same.

4.3. LEMMA. We have $Z_{p, q} \sim\left(\sum \oplus l_{p}^{n}\right)_{(q)}$.

Proof. Put $X=\left(\sum \oplus l_{p}^{n}\right)_{(q)}$. It suffices to show that $Z_{p, q}$ is isomorphic to a complemented subspace of $X$. Then by Lemmas $4.1,4.2$ and Pelczyński's decomposition method we obtain $Z_{p, q} \sim X$. In the following we treat the cases $q \neq 0, \infty$. The proofs for the remaining cases are similar.

Consider $X_{n}:=L_{p}(\partial D)$ endowed with the norm $M_{p}(f, 1) \alpha_{n}^{1 / q}$. Find finite-dimensional subspaces $F_{n} \subset X_{n}$ such that $\left(R_{m_{n}}-R_{m_{n-1}}\right) Z_{p, q} \subset F_{n}$ and $\sup _{n} d\left(F_{n}, l_{p}^{\operatorname{dim} F_{n}}\right)<\infty$. We may identify $X$ with $\left(\sum \oplus F_{n}\right)_{(q)}$. Define $T: Z_{p, q} \rightarrow X$ by $T f=\left(\left(R_{m_{n}}-R_{m_{n-1}}\right) f\right)$. Then $T$ is an isomorphism. Define $S: X \rightarrow Z_{p, q}$ as follows: Each $f_{n} \in F_{n}$ has a natural extension to a harmonic function $\widehat{f}_{n}$ on $D$. So put $S\left(f_{n}\right)=\sum_{n}\left(R_{m_{n}+1}-R_{m_{n-1}-1}\right) \widehat{f}_{n}$. This definition makes sense, at least, if the $f_{n}$ are eventually zero. We have, using (3.1) and (3.2),

$$
\begin{aligned}
& \left\|S\left(f_{n}\right)\right\|_{p, q} \\
& \quad \leq \sum_{k=-2}^{2}\left(\sum_{n} M_{p}^{q}\left(\left(R_{m_{n}}-R_{m_{n-1}}\right)\left(R_{m_{n+k}+1}-R_{m_{n+k-1}-1}\right) \widehat{f}_{n+k}, 1\right) \alpha_{n}\right)^{1 / q} .
\end{aligned}
$$

Recall that $\left\|R_{n}\right\| \leq \lambda_{p}$ for all $n$. By (4.3) we obtain a universal constant $c>0$ such that

$$
\left\|S\left(f_{n}\right)\right\|_{p, q} \leq c\left(\sum_{n} M_{p}^{q}\left(f_{n}, 1\right) \alpha_{n}\right)^{1 / q} .
$$

This means that $S\left(f_{n}\right) \in Z_{p, q}$ and $S$ can be extended to a bounded operator from $X$ to $Z_{p, q}$. By definition and (3.2) we have $S T f=f$ for all $f \in Z_{p, q}$. Hence $T$ is an isomorphism and $T S$ is a bounded projection from $X$ onto $T Z_{p, q} \cdot$

4.4. LemMA. Let $p \in\{1, \infty\}$ and assume that $Y_{p, q} \sim\left(\sum \oplus l_{p}^{n}\right)_{(q)}$. Then $\sup _{n}\left(m_{n}-m_{n-1}\right)<\infty$.

Proof. For a function $f: D \rightarrow \mathbb{C}$ and $\lambda \in \partial D$ put $\left(T_{\lambda} f\right)(z)=f(\lambda z), z \in$ $D$. Fix $n \in \mathbb{Z}$ and for a trigonometric polynomial $f$, let $I_{n} f$ be the trigonometric polynomial with $\left(I_{n} f\right)(w)=w^{n} f(w), w \in \partial D$.

Now assume $\sup _{n}\left(m_{n}-m_{n-1}\right)=\infty$. Fix $\beta>0$ and find, by Lemma 3.2, a trigonometric polynomial $f_{\beta}$ such that $\left\|f_{\beta}\right\|_{p, q}=1$ and $\left\|R f_{\beta}\right\|_{p, q}>\beta$. We can even assume that there are $m_{n-1}, m_{n}$ such that $f_{\beta}$ has the form

$$
f_{\beta}\left(r e^{i \varphi}\right)=\sum_{M \leq|k| \leq N} \gamma_{k} r^{|k|} e^{i k \varphi}
$$


for some $M, N$ with

$$
2^{m_{n-1}+1} \leq M \leq N \leq 3 N \leq 2^{m_{n}} .
$$

(Apply Lemma 3.2 to the indices $m_{n-1}+1$ and $m_{n}-2$.) Put

$$
g_{1}\left(r e^{i \varphi}\right)=\sum_{k=M}^{N} \gamma_{k} r^{k+2 N} e^{i(k+2 N) \varphi}, \quad g_{2}\left(r e^{i \varphi}\right)=\sum_{k=M}^{N} \gamma_{-k} r^{k} e^{i(-k) \varphi}
$$

and

$$
g_{3}\left(r e^{i \varphi}\right)=\left(I_{2 N} g_{2}\right)\left(r e^{i \varphi}\right)=\sum_{k=M}^{N} \gamma_{-k} r^{2 N-k} e^{i(2 N-k) \varphi} .
$$

In view of (4.5) we have, for $j=1,2,3$,

$$
\left(R_{m_{k}}-R_{m_{k-1}}\right) g_{j}= \begin{cases}g_{j}, & k=n \\ 0, & \text { else. }\end{cases}
$$

Moreover, for every $\lambda \in \partial D$,

$$
\left\|T_{\lambda} g_{1}+\lambda^{2 N} I_{2 N} T_{\lambda} g_{2}\right\|_{p, q}=M_{p}\left(T_{\lambda}\left(I_{2 N} f_{\beta}\right), 1\right) \alpha_{n}^{1 / q}=1
$$

and

$$
\left\|g_{1}\right\|_{p, q}=\left\|R f_{\beta}\right\|_{p, q} .
$$

By assumption, Lemma 4.2 provides us with a subspace $X \subset \operatorname{ker} R_{3 N}$ and a projection $Q: Z_{p, q} \rightarrow X$ such that $\|Q\| \leq 2$, and there is a constant $c$ independent of $\beta$ with $d\left(X, Y_{p, q}\right)<c$. Find an isomorphism $T: X \rightarrow Y_{p, q}$ with $\left\|T^{-1}\right\|=1$ and $\|T\|<c$. Fix $\varepsilon>0$. Put $h_{k}\left(r e^{i \varphi}\right)=r^{|k|} e^{i k \varphi}, k \in \mathbb{Z}$. Define

$$
V=\operatorname{span}\left\{h_{-k}: M \leq k \leq N\right\} \text { and } W=X+V .
$$

We obtain $g_{2} \in V$. Extend $T$ to an operator $\widetilde{T}: Y_{p, q}+V \rightarrow W$ by defining

$$
\widetilde{T}(f+g)=T\left(f+I_{2 N} g\right)+\varepsilon g, \quad f \in Y_{p, q}, g \in V .
$$

Since $\left.R_{3 N}\right|_{V}=$ id and $X \subset \operatorname{ker} R_{3 N}$ the operator $\widetilde{T}$ is linear bijective. For $\lambda \in \partial D$ define $S_{\lambda}: W \rightarrow W$ by

(4.8) $\quad S_{\lambda}(\tilde{T} f+\tilde{T} g)=T T_{\lambda} f+\lambda^{2 N} \tilde{T} T_{\lambda} g, \quad f \in Y_{p, q}, g \in V$.

Then $S_{1}=$ id and $S_{\lambda} S_{\mu}=S_{\lambda \mu}$ for all $\lambda, \mu \in \partial D$. Put

$$
\left(Q_{0} h\right)(z)=\frac{1}{2 \pi} \int_{0}^{2 \pi}\left(S_{e^{-i \varphi}} Q S_{e^{i \varphi}} h\right)(z) d \varphi, \quad h \in W, z \in D .
$$

This definitition makes sense since, for fixed $h$, the map $\lambda \mapsto\left(S_{\bar{\lambda}} Q S_{\lambda} h\right)(z)$ is continuous. $Q_{0}$ is a projection from $W$ onto $X$ satisfying $S_{\lambda} Q_{0}=Q_{0} S_{\lambda}$ for all $\lambda$. For $M \leq k \leq N$ we obtain by (4.7), (4.8), since $h_{-k} \in V$,

$$
\lambda^{2 N-k} Q_{0} \tilde{T} h_{-k}=Q_{0} S_{\lambda} \widetilde{T} h_{-k}=S_{\lambda} Q_{0} \widetilde{T} h_{-k} .
$$

Now $\left.R_{3 N}\right|_{X}=0$ and $X \subset Y_{p, q}$ imply that

$$
S_{\lambda} Q_{0} \widetilde{T} h_{-k}=\sum_{j>3 N} \delta_{j} \lambda^{j} h_{j} \quad \text { for some } \delta_{j} \in \mathbb{C} \text { and all } \lambda \in \partial D \text {. }
$$

Hence, by (4.10), $Q_{0} \widetilde{T} h_{-k}=0$ if $M \leq k \leq N$. In particular, $Q_{0} \widetilde{T}\left(g_{1}+g_{2}\right)=$ $T g_{1}$. Thus by (4.6)-(4.8) and the fact that $\left\|S_{\lambda} \mid x\right\| \leq c$,

$$
\begin{aligned}
\beta<\left\|T g_{1}\right\|_{p, q} & \leq \frac{1}{2 \pi} \int_{0}^{2 \pi}\left\|S_{e^{-i \varphi}} Q S_{e^{i \varphi}} \widetilde{T}\left(g_{1}+g_{2}\right)\right\|_{p, q} d \varphi \\
& \leq 2 c \sup _{\lambda \in \partial D}\left\|S_{\lambda} \widetilde{T}\left(g_{1}+g_{2}\right)\right\|_{p, q} \\
& \leq 2 c \sup _{\lambda \in \partial D}\left(\left\|T\left(T_{\lambda} g_{1}+\lambda^{2 N} I_{2 N} T_{\lambda} g_{2}\right)\right\|_{p, q}+\varepsilon\left\|\lambda^{2 N} T_{\lambda} g_{2}\right\|_{p, q}\right) \\
& \leq 2 c^{2}\left(1+\varepsilon\left\|g_{2}\right\|_{p, q}\right) .
\end{aligned}
$$

Since $\varepsilon$ was arbitrarily fixed independent of $\beta$ and $g_{2}$, if $\beta$ is large enough we arrive at a contradiction.

5. Proofs of the main results. First we' go back to Definition 2.4.

5.1. Lemma. Assume that $\mu$ satisfies ( $*$.

(a) There are positive integers $m_{k}$ such that

$$
2 \leq \inf _{k}\left(\frac{\mu_{m_{k-1}}-\mu_{m_{k}}}{\mu_{m_{k}}-\mu_{m_{k+1}}}\right) \leq \sup _{k}\left(\frac{\mu_{m_{k-1}}-\mu_{m_{k}}}{\mu_{m_{k}}-\mu_{m_{k+1}}}\right)<\infty .
$$

(b) If in addition ( $\star \star *)$ is satisfied then there is an integer $K>0$ such that the inequalities of (a) hold for $m_{n}=K n, n=1,2, \ldots$

(c) If $(\star \star)$ is not fulfilled then $\sup _{n}\left(m_{n}-m_{n-1}\right)=\infty$ for any sequence $\left(m_{n}\right)$ of positive integers with $\mu_{m_{n-1}} \geq 3 \mu_{m_{n}}$.

Proof. In (a) we take $m_{1}=1$ and let $m_{k}$ be the smallest integer with $\mu_{m_{k-1}} \geq 3 \mu_{m_{k}}$. If $(\star \star)$ is satisfied then we find $K$ with $\mu_{K n+K} / \mu_{K n} \leq 1 / 3$ for all $n$, and put $m_{n}=K n$. In any case we obtain

Hence

$$
2 \mu_{m_{k}} \leq \mu_{r_{k-1}-1}-\mu_{m_{k}} \leq \mu_{m_{k-1} l} \text { and } \frac{2}{3} \mu_{m_{k}} \leq \mu_{m_{k}}-\mu_{m_{k-1-1}}
$$

$$
2 \leq \frac{\mu_{m_{k_{k-1}-1}}-\mu_{m_{k}}}{\mu_{m_{k}}} \leq \frac{\mu_{m_{k_{k-1}}}-\mu_{m_{k}}}{\mu_{m_{k}}-\mu_{m_{k+1}}} \leq \frac{3}{2} \frac{\mu_{m_{k-1}-1}}{\mu_{m_{k t}}} .
$$

If $m_{n}=K n$ then (a) follows directly from ( $*$ ). If $m_{k}$ is the smallest integer with $3 \mu_{m_{k}} \leq \mu_{m_{k-1}}$ then we have $\mu_{m_{k-1}}<3 \mu_{m_{k}-1}$ and therefore $\mu_{m_{k-1}} / \mu_{m_{k}} \leq 3 \mu_{m_{k}-1} / \mu_{m_{k}}$. We obtain, by $(*)$,

$$
\sup _{k}\left(\frac{\mu_{m_{k-1}}-\mu_{m_{k}}}{\mu_{m_{k}}-\mu_{m_{k-1}}}\right) \leq \frac{9}{2} \sup \left(\frac{\mu_{m_{k}-1}}{\mu_{m_{k}}}\right)<\infty \text {. }
$$

This proves (a) and (b). 
(c) Assume that $\varrho:=2 \sup _{n}\left(m_{n}-m_{n-1}\right)<\infty$ and $\mu_{m_{n-1}} \geq 3 \mu_{m_{n}}$ for all $n$. This implies $3 \mu_{\rho j} \leq \mu_{\rho(j-1)}$ for all $j$. Consider $n$ with $(j-2) \varrho<n \leq$ $\varrho(j-1)$. Then $\varrho j \leq n+2 \varrho \leq \varrho(j+1)$ and we obtain

$$
3 \mu_{n+2 \varrho} \leq 3 \mu_{\varrho j} \leq \mu_{\varrho(j-1)} \leq \mu_{n}
$$

Hence $\mu_{n}$ satisfies $(\star \star)$.

5.2. Proof of Theorem 2.5. Choose $m_{n}$ according to Lemma 5.1. Put $\alpha_{n}=\mu_{m_{n}}-\mu_{m_{n+1}}$ (for $q=0$ or $q=\infty$ we consider $\alpha_{n}=\mu_{m_{n}}$ ). We prove the theorem for $1 \leq q<\infty$. The proof of the remaining cases is similar. (For $p=q=\infty$ see [12].)

Define $r_{n}=1-2^{-m_{n}}$ and $I_{n}=\left[r_{n}, r_{n+1}\left[\right.\right.$. Take $f \in b_{p, q}(\mu)$. Recall that $f_{n}:=\left(R_{m_{n}}-R_{m_{n-1}}\right) f$ is a trigonometric polynomial of degree $\leq 2^{m_{n}+1}$. Hence, by Lemma 3.3 , for $c_{1}=\sup _{n}\left(1-2^{-m_{n}}\right)^{2^{m_{n}+2} q}$ we have $\bar{M}_{p}^{q}(f, 1) \leq$ $c_{1} M_{p}^{q}\left(f, r_{n}\right)$ and thus

$$
\frac{1}{c_{1}} M_{p}^{q}\left(f_{n}, 1\right) \alpha_{n} \leq \int_{I_{n}} M_{p}^{q}\left(f_{n}, r\right) d \mu(r) \leq M_{p}^{q}\left(f_{n}, 1\right) \alpha_{n}
$$

Similarly, by Lemma 3.3, there is a universal constant $c_{2} \geq c_{1}$ with

$$
M_{p}\left(f_{j}, r_{n+1}\right) \alpha_{n}^{1 / q} \leq c_{2} \begin{cases}\left(\alpha_{n} / \alpha_{j}\right)^{1 / q} M_{p}\left(f_{j}, 1\right) \alpha_{j}^{1 / q}, & j \leq n \\ r_{n+1}^{2^{m_{j-1}}}\left(\alpha_{n} / \alpha_{j}\right)^{1 / q} M_{p}\left(f_{j}, 1\right) \alpha_{j}^{1 / q}, & j>n\end{cases}
$$

Put

$$
\beta_{n, j}=\left\{\begin{array}{ll}
(1 / 2)^{(n-j) / q}, & j \leq n, \\
\exp \left(-2^{m_{j-1}-m_{n+1}}\right) \varrho^{(j-n) / q}, & j>n,
\end{array} \quad \text { for } \varrho=\sup _{k}\left(\alpha_{k-1} / \alpha_{k}\right)\right.
$$

Using Lemma 5.1 we obtain a constant $c_{3} \geq c_{2}$ with $M_{p}\left(f_{j}, r_{n+1}\right) \alpha_{n}^{1 / q} \leq$ $c_{3} \beta_{n, j} M_{p}\left(f_{j}, 1\right) \alpha_{j}^{1 / q}$. Note that $\sup _{n} \sum_{j=1}^{\infty} \beta_{n, j}<\infty$. The Hölder inequality yields

$$
\begin{aligned}
\|f\|_{p, q} & \leq\left(\sum_{n} \int_{I_{n}}\left(\sum_{j} M_{p}\left(f_{j}, r\right)\right)^{q} d \mu(r)\right)^{1 / q} \\
& \leq\left(\sum_{n}\left(\sum_{j} M_{p}\left(f_{j}, r_{n+1}\right) \alpha_{n}^{1 / q}\right)^{q}\right)^{1 / q} \\
& \leq c_{3}\left(\sum_{n}\left(\sum_{j} \beta_{n, j} M_{p}\left(f_{j}, 1\right) \alpha_{j}^{1 / q}\right)^{q}\right)^{1 / q} \\
& \leq c_{4}\left(\sum_{n} \sum_{j} \beta_{n, j} M_{p}^{q}\left(f_{j}, 1\right) \alpha_{j}\right)^{1 / q}
\end{aligned}
$$

$$
\begin{aligned}
= & c_{4}\left(\sum _ { j } \left(\sum_{n=j}^{\infty}(1 / 2)^{(n-j) / q}\right.\right. \\
& \left.\left.+\sum_{n=1}^{j-1} \exp \left(-2^{m_{j-1}-m_{n+1}}\right) \varrho^{(j-n) / q}\right) M_{p}^{q}\left(f_{j}, 1\right) \alpha_{j}\right)^{1 / q} \\
\leq & c_{5}\left(\sum_{j} M_{p}^{q}\left(f_{j}, 1\right) \alpha_{j}\right)^{1 / q}
\end{aligned}
$$

for universal constants $c_{5} \geq c_{4} \geq c_{3}$.

Conversely, according to $(5.1)$ and (1.3),

$$
\begin{aligned}
c_{1}^{-1 / q}\left(\sum_{j=1}^{\infty} M_{p}^{q}\left(f_{j}, 1\right) \alpha_{j}\right)^{1 / q} & \\
& \leq\left(\sum_{j=1}^{\infty} \int_{I_{j}} M_{p}^{q}\left(f_{j}, r\right) d \mu(r)\right)^{1 / q} \\
& \leq 2 \lambda_{p}\left(\sum_{j=1}^{\infty} \int_{I_{j}} M_{p}^{q}(f, r) d \mu(r)\right)^{1 / q} \leq 2 \lambda_{p}\|f\|_{p, q}
\end{aligned}
$$

If $(\star \star)$ is satisfied then we find $K$ such that we can choose $m_{n}=K n$ in the preceding estimates. In this case we have

$$
R_{K n}-R_{K n-K}=\sum_{j=1}^{K}\left(R_{K(n-1)+j}-R_{K(n-1)+j-1}\right)
$$

Thus according to Lemma 3.1 , if $p \in\{1, \infty\}$, the Riesz projection $R$ : $b_{p, q}(\mu) \rightarrow B_{p, q}(\mu)$ is bounded with respect to $\|\cdot\|_{p, q}$.

If $(* \star)$ is not satisfied then, by Lemma 5.1 , our choice of $m_{n}$ implies $\sup _{n}\left(m_{n}-m_{n-1}\right)=\infty$. If $p \in\{1, \infty\}$, then Lemma 3.2 shows that $R$ is unbounded. Hence for no choice of $\left(m_{n}\right)$ such that the first part of Theorem 2.5 holds can we have $\sup _{n}\left(m_{n}-m_{n-1}\right)<\infty$.

5.3. Proof of Corollary 2.6. Theorem 2.5 shows that $b_{p, q}(\mu) \sim$ $Z_{p, q}$ with $\alpha_{n l}=\mu_{m_{n h}}-\mu_{m_{n-1+1}}\left(\alpha_{n}=\mu_{n_{n}}\right.$ if $\left.q \in\{0, \infty\}\right)$. (4.3) is satisfied according to Lemma 5.1. So (a) follows from Lemma 4.3.

Moreover, we have $B_{p, q}(\mu) \sim Y_{p, q}$. Let $1<p<\infty$. Then the Riesz projection is always bounded with respect to $\|\cdot\|_{p, q}$ because $R$ is bounded with respect to $M_{p}(\cdot, 1)$, Hence $B_{p, q}(\mu)$ is complemented in $b_{p, q}(\mu)$ if $1<$ $p<\infty$. Now, Lernmas 4.1 and 4.2 together with Pelczyński's decomposition method show that $B_{p, q}(\mu) \sim\left(\sum \Theta l_{p}^{n}\right)_{(q)}$. This proves Corollary 2.6(b).

Since $R$, is bounded, in view of $(2.6)$, the map $f \mapsto \widetilde{f}$ is bounded with respect to $\|\cdot\|_{p, q}$. This yields (d). 
5.4. Proof of Corollary 2.7. (ii) $\Leftrightarrow$ (iv) follows directly from (2.6) since $R$ is bounded with respect to $\|\cdot\|_{p, q}$ if and only if the conjugation operator is bounded. Theorem 2.5 in connection with Lemmas 3.1 and 3.2 shows (ii) $\Leftrightarrow$ (iii).

If $R$ is bounded then $B_{p, q}(\mu) \sim Y_{p, q}$ is complemented in $b_{p, q}(\mu) \sim$ $Z_{p, q}$. Lemmas 4.1 and 4.2 and an application of Pełczyński's decomposition method yield $B_{p, q}(\mu) \sim\left(\sum \oplus l_{p}^{n}\right)_{(q)}$.

Finally, if $B_{p, q}(\mu) \sim\left(\sum \oplus l_{p}^{n}\right)_{(q)}$ then Lemma 4.4 implies $\sup _{n}\left(m_{n}-\right.$ $\left.m_{n-1}\right)<\infty$. So, by Lemma $3.1, R$ is bounded.

\section{References}

[1] S. Axler, Bergman spaces and their operators, in: Survey of Some Recent Results in Operator Theory, B. Conway and B. Morrel (eds.), Pitman Res. Notes, 1988, $1-50$.

[2] K. D. Bierstedt and W. H. Summers, Biduals of weighted Banach spaces of analytic functions, J. Austral. Math. Soc. Sec. A 54 (1993), 70-79.

[3] O. Blasco, Multipliers on weighted Besov spaces of analytic functions, in: Contemp. Math. 144, Amer. Math. Soc., 1993, 23-33.

[4] R. R. Coifman and R. Rochberg, Representation theorems for holomorphic and harmonic functions in $L^{p}$, Astérisque 77 (1980), 12-66.

[5] P. L. Duren, Theory of $H^{p}$-Spaces, Academic Press, New York, 1970.

[6] T. M. Flett, The dual of an inequality of Hardy and Littlewood and some related inequalities, J. Math. Anal. Appl. 38 (1972), 746-765.

[7] - Lipschitz spaces of functions on the circle and the disc, ibid. 39 (1972), 125-158.

[8] G. H. Hardy and J. E. Littlewood, Some properties of fractional integrals $I I$, Math. Z. 34 (1932), 403-439.

[9] -, -, Theorems concerning mean values of analytic or harmonic functions, Quart. J. Math. 12 (1941), 221-256.

[10] J. Lindenstrauss and A. Pelczyński, Contributions to the theory of classical Banach spaces, J. Funct. Anal. 8 (1971), 225-249.

[11] W. Lusky, On the structure of $H v_{0}(D)$ and $h v_{0}(D)$, Math. Nachr. 159 (1992), 279-289.

[12] -, On weighted spaces of harmonic and holomorphic functions, J. London Math. Soc. (2) 51 (1995), 309-320.

[13] M. Mateljević and M. Pavlović, $L^{p}$-behaviour of the integral means of analytic functions, Studia Math. 77 (1984), 219-237.

[14] L. A. Rubel and A. L. Shields, The second duals of certain spaces of analytic, functions, J. Austral. Math. Soc. 11 (1970), 276-280.

[15] A. L. Shields and D. L. Williams, Bounded projections, duality and multipliers in spaces of analytic functions, Trans. Amer. Math. Soc. 162 (1971), 287-302.

[16] - - , Bounded projections, duality and multipliers in spaces of harmonic functions, J. Reine Angew. Math. 299/300 (1978), 256-279.

[17] - - - Bounded projections and the growth of harmonic conjugates in the unit disc, Michigan Math. J. 29 (1982), 3-25.
[18] A. Torchinsky, Real-Variable Methods in Harmonic Analysis, Academic Press, New York, 1986.

[19] P. Wojtaszczyk, Banach Spaces for Analysts, Cambridge University Press, 1991.

[20] -, On unconditional polynomial bases in $L_{p}$ and Bergman spaces, Constr. Approx., to appear.

\section{Fachbereich 17}

Universität-Gesamthochschule

Warburger Straße 100

D-33098 Paderborn, Germany

E-mail: lusky@uni-paderborn.de 